indicate that one cycle of treatment is sufficient to expand and generate de novo clones (figure 2). We developed a primary cell cytotoxicity assay and observe that cytotoxic potency is target dependent. Target cell killing and T cell activation/ proliferation depend on CD33 clustering, and both CD4 and CD8 $\mathrm{T}$ cells can engage and kill target cells. This is illustrated in assays with KG-1 (M2, clustered) and KG-1a (M0, not clustered) cell lines, in which the KG-1 cells have an EC50 15-20 fold lower than the M0 cell line (figure 3). In addition, there is little to no detectable binding or killing of monocytes or neutrophils, which is consistent with the absence of neutropenia in patients enrolled in the trial to date.

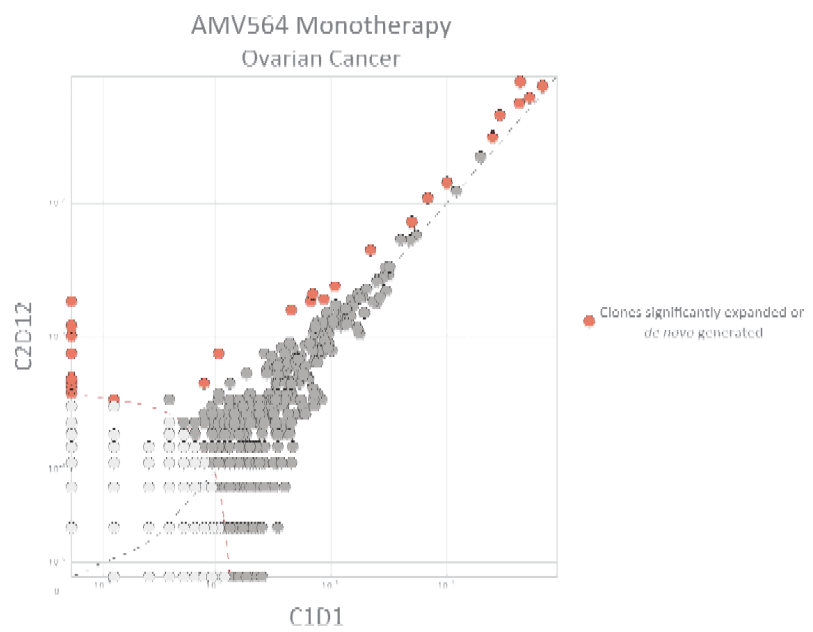

Abstract 692 Figure 2 TcRb CDR3 sequencing of an ovarian cancer patient shows extensive clonal expansion upon treatment. Scatter plots represent clonal abundance in the periphery between Baseline (C1D1) and C1D12 using the differential Abundance tool (Adaptive Biotechnology)

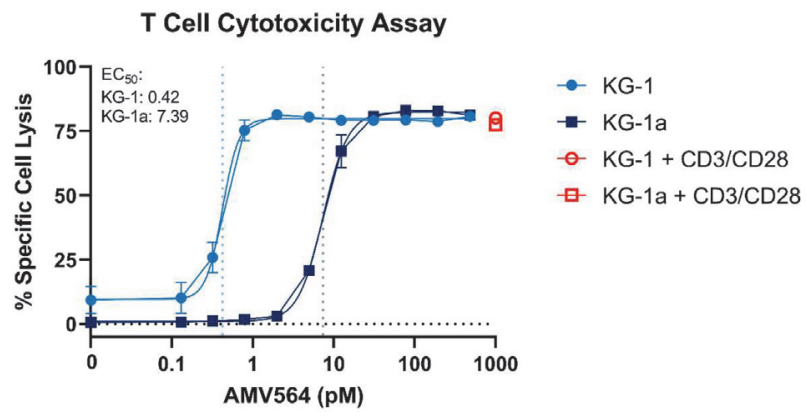

Abstract 692 Figure 3 Cytotoxicity assay using fresh primary T cells demonstrates target selectivity on two cell lines with equivalent CD33 surface expression. T cell activation by AMV564 at clinically relevant doses is equivalent to $\mathrm{CD} 3 / \mathrm{CD} 28$ stimulation

Conclusions AMV564 is a potent conditional $\mathrm{T}$ cell agonist which is clinically active. We demonstrate that the combination of $\mathrm{T}$ cell activation, increased $\mathrm{T}$ cell diversity, and target specificity allow AMV564 to deplete MDSCs and restore a native immune response to cancer.

Ethics Approval This study was approved by the Institutional Review Board (IRB) or Independent Ethics Committee (IEC) at each participating institution.

http://dx.doi.org/10.1136/jitc-2020-SITC2020.0692
693 APX601, A NOVEL TNFR2 ANTAGONIST ANTIBODY FOR CANCER IMMUNOTHERAPY

Erin Filbert*, Sushma Krishnan, Ryan Alvarado, George Huang, Francis Bahjat, Xiaodong Yang. Apexigen, Inc., San Carlos, CA, USA

Background A key barrier to effective immunotherapy for cancer is the immunosuppressive tumor microenvironment (TME) characterized by infiltrating regulatory $\mathrm{T}$ cells (Tregs) and myeloid-derived suppressor cells (MDSC). While depletion of immune-suppressive cells is a promising cancer immunotherapy strategy, current approaches are ineffective due to lack of specificity and safety concerns. Tumor Necrosis Factor Receptor 2 (TNFR2) is emerging as a novel, selective target to overcome immunosuppression in TME. TNFR2 expression is generally restricted to highly immunosuppressive cell populations in the TME and the TNFR2-TNF- $\alpha$ pathway plays an important role in the generation and survival of these cells. TNFR2 is also an oncogene upregulated on certain tumors and can enhance tumor cell survival. Thus, targeting TNFR2 is a promising therapeutic approach with multiple potential mechanisms of action.

Methods A diverse panel of antibodies to TNFR2 was created using $\mathrm{APXiMAB}^{\mathrm{TM}}$, Apexigen's proprietary rabbit monoclonal antibody technology. A robust assessment of over 100 antibody candidates for TNFR2 binding, TNF- $\alpha$ blockade and functional assays yielded APX601, a humanized IgG1 antibody, as the lead therapeutic candidate. The ability of APX601 to reverse immune suppression was assessed in Treg and MDSC suppression assays. In addition, the ability of APX601 to deplete TNFR2-expressing Treg and tumor cells was assessed both in vitro and in vivo using the mouse Colo205 xenograft model.

Results APX601 binds specifically to human TNFR2 with high affinity $(\mathrm{Kd}=47 \mathrm{pM})$ and recognizes a unique epitope in the CRD1 domain of TNFR2. APX601 is a potent antagonist that blocks the TNFR2-TNF- $\alpha$ interaction in cell-based ligand binding assays (IC50 $=0.149 \mathrm{nM}$ ). APX601 is capable of reversing immune suppression via two mechanisms: 1) significant blockade of the immunosuppressive functions of both Tregs and MDSCs by inhibiting the binding of TNFR2 to its ligand TNF- $\alpha$ and 2) depletion of TNFR2-expressing Tregs, MDSC and tumor cells via antibody-dependent cell cytotoxicity (ADCC) $(\mathrm{EC50}=1.14$ $\mathrm{nM})$ and ADCP (EC50 $=0.71 \mathrm{nM})$ effector functions.

Conclusions APX601 is a potent TNFR2 antagonist antibody that reverses immune suppression by targeting TNFR2-expressing Treg and MDSC, and induces killing of tumor cells. Our data support the further development of APX601, a promising immunotherapeutic antibody with multiple potential mechanisms of action, for the treatment of a variety of solid tumors.

Ethics Approval Healthy human blood samples were obtained from Stanford Blood Center (Palo Alto, CA) from consenting donors under an approved protocol.

http://dx.doi.org/10.1136/jitc-2020-SITC2020.0693

\section{NC410 IS A NOVEL IMMUNOMEDICINE FOR THE TREATMENT OF SOLID TUMORS}

${ }^{1}$ Linjie Tian, ${ }^{2} \mathrm{M}$ Ines Pascoal Ramos, ${ }^{2}$ Emma de Ruiter, ${ }^{1}$ Ana Paucarmayta, ${ }^{2}$ Eline Elshof, ${ }^{2}$ Stefan Willems, ${ }^{1}$ Chang Song, 'Zachary Cusumano, 'Jason Bosiacki, 'Linda Liu, ${ }^{1}$ Solomon Langermann, ${ }^{2}$ Linde Meyaard, 'Dallas Flies*. 'NextCure, Inc., Beltsville, MD, USA; ${ }^{2}$ University Medical Center, Utrecht, Utrecht, Netherlands

Background Abnormalities in the extracellular matrix of tumor microenvironments support tumor progression, lead to 
immune dysfunction, and provide a target for cancer therapeutics. Collagens are a primary component of the extracellular matrix. Abnormal levels of collagen and of the collagendomain containing complement component $1 \mathrm{q}(\mathrm{C} 1 \mathrm{q})$ in tumor microenvironments has been proposed to disrupt anti-tumor immunity. LAIR-1 is an adhesion molecule and inhibitory receptor expressed on the cell surface of several immune cell subsets. LAIR-1 binding to collagen-like domains present in collagens and C1q inhibit immune cell function. LAIR-2 is a soluble homolog of LAIR-1 that binds to and outcompetes LAIR-1 binding to collagens and $\mathrm{C} 1 \mathrm{q}$ and serves as a natural decoy to promote immune function.

Methods Taking advantage of a natural decoy system, we designed a protein biologic, NC410, composed of LAIR-2 fused with a functional IgG1 Fc domain to target collagenrich tumors and promote immune activation, infiltration and effector function.

Results NC410 has increased avidity due to Fc mediated dimerization, and blocks LAIR-1 interactions with ligands, and LAIR-1 signaling. In vivo administration of NC410 in humanized tumor models reduced tumor growth in a dose dependent fashion. NC410 increased the numbers of infiltrating human $\mathrm{CD} 8+$ and CD4 $+\mathrm{T}$ cells in the tumor, which is associated with increased levels of chemokines in the local tumor environment. Effector function was also enhanced, as denoted by increased levels of IFN-gamma and Granzyme B in the local tumor environment. In addition, NC410 increased specific collagen degradative products in the serum of humanized tumor-bearing mice, suggesting NC410 may promote tumor microenvironment remodeling and immune accessibility to further promote anti-tumor immunity.

Conclusions These data support NC410 as a novel therapeutic for targeting collagen-rich tumors and enabling normalization of the tumor-immune microenvironment. FIH studies have recently been initiated with NC410.

http://dx.doi.org/10.1136/jitc-2020-SITC2020.0694

\section{ORAL DELIVERY OF A MICROBIAL EXTRACELLULAR VESICLE INDUCES POTENT ANTI-TUMOR IMMUNITY IN MICE}

Loise Francisco-Anderson, Loise Francisco-Anderson, Mary Abdou, Michael Goldberg, Erin Troy, Alicia Ballok, Fabian Romano Chernac, Maria Sizova, Krutika Invally, Shubhra Kashyap, Audrey McBride, Jessica Tsang, Shannon Argueta, Kristie Barth, Valeria Kravitz, Holly Ponichtera, Tyler Rommel, Kevin Huynh, Tanmoy Ganguly, Mark Bodmer*, Mark Bodmer. Evelo Biosciences, Cambridge, MA, USA

Background The small intestinal axis (SINTAX) is a network of anatomic and functional connections between the small intestine and the rest of the body. It acts as an immunosurveillance system, integrating signals from the environment that affect physiological processes throughout the body. The impact of events in the gut in the control of tumor immunity is beginning to be appreciated. We have previously shown that an orally delivered single strain of commensal bacteria induces anti-tumor immunity preclinically via pattern recognition receptor-mediated activation of innate and adaptive immunity. Some bacteria produce extracellular vesicles (EVs) that share molecular content with the parent bacterium in a particle that is roughly $1 / 1000$ th the volume in a non-replicating form. We report here an orally-delivered and gut-restricted bacterial EV which potently attenuates tumor growth to a greater extent than whole bacteria or checkpoint inhibition.
Methods EDP1908 is a preparation of extracellular vesicles produced by a gram-stain negative strain of bacterium of the Oscillospiraceae family isolated from a human donor. EDP1908 was selected for its immunostimulatory profile in a screen of EVs from a range of distinct microbial strains. Its mechanism of action was determined by ex vivo analysis of the tumor microenvironment (TME) and by in vitro functional studies with murine and human cells.

Results Oral treatment of tumor-bearing mice with EDP1908 shows superior control of tumor growth compared to checkpoint inhibition (anti-PD-1) or an intact microbe. EDP1908 significantly increased the percentage of IFN $\gamma$ and TNF producing CD8 + CTLs, NK cells, NKT cells and CD4+ cells in the tumor microenvironment (TME). EDP1908 also increased tumor-infiltrating dendritic cells (DC1 and DC2). Analysis of cytokines in the TME showed significant increases in IP-10 and IFNg production in mice treated with EDP1908, creating an environment conducive to the recruitment and activation of anti-tumor lymphocytes.

Conclusions This is the first report of striking anti-tumor effects of an orally delivered microbial extracellular vesicle. These data point to oral EVs as a new class of immunotherapeutic drugs. They are particularly effective at harnessing the biology of the small intestinal axis, acting locally on host cells in the gut to control distal immune responses within the TME. EDP1908 is in preclinical development for the treatment of cancer.

Ethics Approval Preclinical murine studies were conducted under the approval of the Avastus Preclinical Services' Ethics Board. Human in vitro samples were attained by approval of the IntegReview Ethics Board; informed consent was obtained from all subjects.

http://dx.doi.org/10.1136/jitc-2020-SITC2020.0695

\section{BRENTUXIMAB VEDOTIN, A CD30-DIRECTED ANTIBODY- DRUG CONJUGATE, SELECTIVELY DEPLETES ACTIVATED TREGS IN VITRO AND IN VIVO}

Bryan Grogan*, Reice James, Michelle Ulrich, Shyra Gardai, Ryan Heiser, Reice James Seattle Genetics, Inc., Bothell, WA, USA

Background Regulatory $\mathrm{T}$ cells (Tregs) play an important role in maintaining immune homeostasis, preventing excessive inflammation in normal tissues. In cancer, Tregs hamper antitumor immunosurveillance and facilitate immune evasion. Selective targeting of intratumoral Tregs is a potentially promising treatment approach. Orthogonal evaluation of tumorinfiltrating lymphocytes (TILs) in solid tumors in mice and humans have identified CCR8, and several tumor necrosis family receptors (TNFRs), including TNFSFR8 (CD30), as receptors differentially upregulated on intratumoral Tregs compared to normal tissue Tregs and other intratumoral $\mathrm{T}$ cells, making these intriguing therapeutic targets.Brentuximab vedotin (BV) is approved for classical Hodgkin lymphoma (cHL) across multiple lines of therapy including frontline use in stage III/IV cHL in combination with doxorubicin, vinblastine, and dacarbazine. BV is also approved for certain CD30-expressing T-cell lymphomas. BV is comprised of a CD30-directed monoclonal antibody conjugated to the highly potent microtubuledisrupting agent monomethyl auristatin E (MMAE).The activity of $\mathrm{BV}$ in lymphomas is thought to primarily result from tumor directed intracellular MMAE release, leading to mitotic arrest and apoptotic cell death.The role CD30 plays in normal 